\title{
Critical role of the Toll-like receptor signal adaptor protein MyD88 in acute allograft rejection
}

\author{
Daniel R. Goldstein, ${ }^{1}$ Bethany M. Tesar, ${ }^{1}$ Shizuo Akira, ${ }^{2}$ and Fadi G. Lakkis ${ }^{3}$ \\ ${ }^{1}$ Section of Cardiovascular Medicine, Department of Internal Medicine, Yale University School of Medicine, \\ New Haven, Connecticut, USA \\ ${ }^{2}$ Department of Host Defense, Research Institute for Microbial Diseases, Osaka University, Osaka, Japan \\ ${ }^{3}$ Section of Nephrology, Department of Internal Medicine, and Section of Immunobiology, Yale University School of Medicine, \\ New Haven, Connecticut, USA
}

\begin{abstract}
The Toll-like receptors (TLRs) are recently discovered germline-encoded receptors on APCs that are critically important in innate immune recognition of microbial pathogens. However, their role in solid-organ transplantation is unknown. To explore this role, we employed a skin allograft model using mice with targeted deletion of the universal TLR signal adaptor protein, MyD88. We report that minor antigen-mismatched (HY-mismatched) allograft rejection cannot occur in the absence of MyD88 signaling. Furthermore, we show that the inability to reject these allografts results from a reduced number of mature DCs in draining lymph nodes, leading to impaired generation of anti-graft-reactive $T$ cells and impaired Th1 immunity. Hence, this work demonstrates that TLRs can be activated in a transplant setting and not solely by infections. These results link innate immunity to the initiation of the adaptive alloimmune response.
\end{abstract}

J. Clin. Invest. 111:1571-1578 (2003). doi:10.1172/JCI200317573.

\section{Introduction}

The recently discovered Toll-like receptors (TLRs) are germline-encoded, transmembrane receptors that are critical for the detection of microbial pathogens (1-3). It has been postulated that invading microbes express pathogen-associated molecular patterns (PAMPs) that stimulate host pathogen recognition receptors (PRRs) (4). TLRs are a specific form of PRR that are expressed on the surface of APCs. Gram-negative sepsis is an example of a PAMP-PRR interaction in which LPS on the invading pathogen stimulates TLR4, which acts as a heterodimer with CD14 and MD2 on host APCs, initiating an inflammatory response that is responsible for septic shock $(3,5)$. Another prominent TLR is TLR2, which, by forming heterodimers with TLR1 and TLR6, can recognize a broad range of pathogens, including peptidoglycan on Gram-positive bacteria, bacterial lipoproteins, and glycosylphosphatidylinositol lipids from Trypanosoma cruzi (6).

Once ligated, TLRs initiate a signaling pathway via their universal signal adaptor protein, MyD88 (7), inducing the translocation of NF- $\mathrm{KB}$, which in DCs and other APCs initiates a maturation program consisting

Received for publication December 9, 2002, and accepted in revised form March 19, 2003.

Address correspondence to: Daniel R. Goldstein, Section of Cardiovascular Medicine, Yale University School of Medicine, 135 College Street, Suite 301, New Haven, Connecticut 06510, USA. Phone: (203) 785-2917; Fax: (203) 737-1801;

E-mail: daniel.goldstein@yale.edu.

Conflict of interest: The authors have declared that no conflict of interest exists.

Nonstandard abbreviations used: Toll-like receptor (TLR); pathogen-associated molecular pattern (PAMP); pathogen recognition receptor (PRR); median survival time (MST). of increased expression of costimulatory molecules and release of proinflammatory cytokines (6). This allows DCs to mature and migrate to the draining lymph nodes and to initiate an immune response by activating naive $T$ cells. This pathway has been shown to be critical for Th1 immunity to microbial antigens (7). Hence, TLR signaling is a major mechanism by which the immune system can distinguish self from microbial non-self and control adaptive immunity (4). These discoveries are consistent with the hypothesis that mammalian immune systems evolved to fight off invading pathogens $(4,8,9)$. However, the role of TLRs in allograft rejection is unknown. Several studies have shown that TLRs can be activated by endogenous ligands like heat shock protein, heparan sulfate, surfactant, and the contents of necrotic cells (10-13). Other studies have demonstrated increased expression of some of these ligands during allograft rejection (14). Therefore, we thought it plausible that TLR signaling may be activated in the setting of organ transplantation, leading to an adaptive alloimmune response. To test this hypothesis, we employed a skin allograft model using mice with targeted mutations of TLR2, TLR4, or the MyD88 adaptor protein. Specifically, we used a minor antigen-mismatched (HY-mismatched) model to control for TLR and $M y D 88$ expression in both the recipient and the graft. Because IL-1 and IL-18 also signal via MyD88 (15) and are both synthesized in an inactive form that requires cleavage via caspase-1 (16), we used mice deficient in IL-1 $\beta$-converting enzyme and caspase- 1 to control for TLR-independent MyD88 signaling in our model. We present evidence here that the alloimmune response to an allograft is critically dependent on signaling via MyD88. 


\section{Methods}

Mice and skin transplantation. Full-thickness trunk skin was harvested from 6- to 8-week-old donor mice. The recipient graft area and donor skin were prepared by cleaning with Betadine and $70 \%$ ethanol. The grafts were sutured with staple clips on the left thorax of 6- to 8 -week-old recipients as previously described (17). In addition, allografts were impregnated with antibiotic ointment. Rejection was defined as graft necrosis greater than $90 \%$ of graft area. B6;129/SvJ-MyD88 ${ }^{\text {tmlAKI }}$ mice (referred to as MyD88-/-) were generously provided by Shizuo Akira (Osaka University). The mice were derived from segregated B6;129/SvJ background mice. Heterozygous mice were maintained to obtain homozygous mice (MyD88-/-) and WT littermate controls $\left(\mathrm{MyD}^{+/+}\right.$). B6;129/SvJ-TLR2 ${ }^{\text {tmlAKI }}$ (referred to as TLR2 $^{-/}$) and B6;129/SvJ-TLR4 ${ }^{\text {tmlaKI }}$ (referred to as TLR4 $^{-/}$) mice were also generously provided by Shizuo Akira. B6;129/SvJ-Casp $1^{\mathrm{tm} 1 \mathrm{FLV}}$ mice (referred to as $\mathrm{ICE}^{-/-}$) were generously provided by Ruslan Medzhitov (Yale University). C57BL/10AiTac-TgN(HY TCR) N12 transgenic mice (referred to as $\mathrm{HY}$ transgenic) were purchased from Taconic (Germantown, New York, USA). All mice were housed in pathogen-free conditions, and animal protocols were approved by Yale University Institutional Animal Care and Use Committee.

In vivo activation and spleen cell preparation prior to adoptive transfer. Female mice were injected intraperitoneally with $2 \times 10^{7}$ male spleen cells. Seven days later, female spleen cells were harvested in PBS and subjected to red blood cell lysis using a hypotonic solution. At the time of transplantation, $4 \times 10^{7}$ spleen cells were infused per recipient via tail vein injection.

Cell preparation and flow cytometric analysis. To analyze DC phenotype, cells were harvested from lymph nodes draining the skin grafts (superficial axillary and inguinal nodes pooled from three mice per group) on days 0,7 , and 14 post-transplantation. Cells were then stained with the following mAb's (all obtained from Pharmingen, San Diego, California, USA): FITC-conjugated rat anti-mouse CD80, -mouse CD86, and -mouse CD40, and phycoerythrin-conjugated rat antimouse CD11c. Incubation lasted 30 minutes at $4^{\circ} \mathrm{C}$. Isotype control antibodies were used in every experiment. To reduce any confounding DC maturation by contaminating LPS, polymyxin was incorporated, since it has been shown to inhibit LPS at $20 \mu \mathrm{g} / \mathrm{ml}$ (13). We confirmed this in a dose-titration experiment (data not shown). To analyze graft-reactive CD8 T cells on day 14 after transplantation, spleen cells were first $T$ cell-enriched using magnetic microbeads conjugated to rat anti-mouse Thy1.2 (Miltenyi Biotec Inc., Auburn, California, USA). Single-cell suspension was incubated on ice for 15 minutes with $10 \mu \mathrm{l}$ of microbeads per $10^{7}$ cells, washed, and then run on an automated magnetic cell sorter cell-separating system (autoMACS; Miltenyi Biotec Inc.) (>90\% purity). The purified T cells were then stained with phycoerythrin-conjugated antiHY tetramers (KCSRNRQYL; a gift from the tetramer facility of the National Institute of Allergy and Infectious Diseases, Bethesda, Maryland, USA) and FITCconjugated anti-CD8 (Pharmingen). Phycoerythrinconjugated anti-lymphocytic choriomeningitis virus (KAVYNFATC) was purchased from Beckman Coulter Inc. (San Diego, California, USA). Tetramers were used at a 1:100 dilution (shown to give the most specific staining in a dose-titration experiment) and incubated at $4^{\circ} \mathrm{C}$ for 1 hour. All analyses were performed on a FACSCalibur flow cytometer using CellQuest software (Becton Dickinson Immunocytometry Systems, San Diego, California, USA), and all experiments were repeated in triplicate.

RNA isolation and gene expression by real-time PCR. RNA was isolated from draining lymph nodes using RNeasy Mini Kits (QIAGEN Inc., Valencia, California, USA) according to the manufacturer's recommendations. Reverse transcription to cDNA was performed using reverse transcription mastermix (GIBCO BRL; Life Technologies Inc., Carlsbad, California, USA) according to the manufacturer's instructions. Real-time PCR was performed using TaqMan primers and probes for IFN- $\gamma$ and IL-4, all purchased from Applied Biosystems (Foster City, California, USA) and used according to the manufacturer's instructions with an ABI 7700 thermocycler (Applied Biosystems). Data were collected with Sequence Detector software (Applied Biosystems) from which an amplification plot was generated. From this plot the threshold value (CT) was calculated, representing the PCR cycle value at which fluorescence was detectable above an arbitrary threshold. Relative gene expression between naive and transplanted animals within each group was calculated using the $\Delta \Delta \mathrm{CT}$ method $(18,19)$. The amount of cDNA loaded into wells was controlled by measurement of CT values for ribosomal 18S RNA. All experiments were repeated in triplicate.

Statistical analysis. Allograft-survival comparisons between groups were analyzed using the log rank method. Comparison of means was performed using a two-tailed $t$ test and repeated-measures ANOVA. All results were generated using SPSS statistical software (SPSS Science, Chicago, Illinois, USA). Differences were considered statistically significant if $P$ was less than 0.05 .

\section{Results}

Rejection of HY-incompatible skin allografts is dependent on the MyD88 pathway. We used a skin allograft model to test the hypothesis that TLRs are critically important in transplant rejection. As allograft rejection can be initiated by either donor passenger APCs or recipient APCs (20), we used an HY-incompatible transplant model whereby we could control for expression and function of TLRs and MyD88 in both the graft and the recipient. Therefore, to determine whether TLR signaling is important for allograft rejection, female mice with targeted deletions of both copies of TLR2, TLR4, or MyD88 genes (B6;129/SvJ background, $\mathrm{H} 2^{\mathrm{b}}$ ) were transplanted with full-thickness male skin 


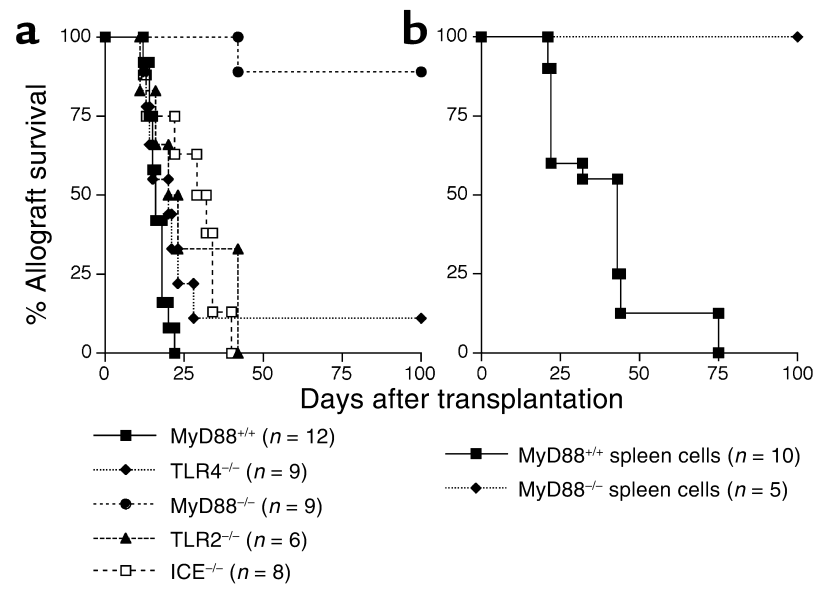

allografts from corresponding littermate geneknockout donors (i.e., skin grafts from mutant male donors transplanted onto mutant female recipients). To control for the IL-1 and IL-18 pathways, female caspase-1 knockout recipients (ICE ${ }^{-/-}$) were grafted with male $\mathrm{ICE}^{-/-}$allografts. Genotypes were confirmed by PCR (data not shown), and all recipients received syngeneic transplants as technical controls. The results demonstrate that eight out of nine

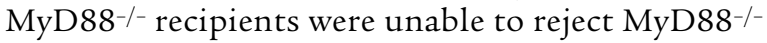
male allografts (median survival time [MST] > 100 days) (Figure 1a). In contrast, all WT littermate controls rejected their allografts (MST $=16$ days, $P=0.00001)$ (Figure 1a). TLR2 $2^{-/}$and $\mathrm{ICE}^{-/-}$recipients had significant but small prolongation of their allograft survival time compared with WT littermate controls $\left(\right.$ TLR2 $^{-/-}$MST $=21$ days, $P=0.02$ vs. WT littermate controls; $\mathrm{ICE}^{-/-} \mathrm{MST}=32$ days, $P=0.01$ vs. WT littermate controls) (Figure 1a). Importantly, allograft survival in both the TLR2-/- and the $\mathrm{ICE}^{-/-}$recipients

\section{Figure 2}

MyD88-1- recipients have a reduced number of mature and immature DCs in lymph nodes draining the allografts. (a) Despite having similar numbers of mature $\left(\mathrm{CD} 40^{+} \mathrm{CD} 11 \mathrm{c}^{+}, \mathrm{CD} 80^{+} \mathrm{CD} 11 \mathrm{c}^{+}\right.$, and $\left.\mathrm{CD} 86^{+} \mathrm{CD} 11 \mathrm{c}^{+}\right) \mathrm{DC}$ on day $0, \mathrm{MyD} 88^{-/-}$recipients were unable to increase numbers of mature DCs on days 7 and 14, whereas WT counterparts had stable numbers of mature DCs during the first week that increased nearly fivefold by day $14(P=0.0001$ vs.

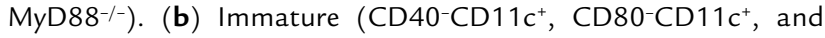
CD86-CD11 $\mathrm{c}^{+}$) DCs failed to accumulate in the draining lymph nodes of allografts of MyD88 $/ /$ recipients of MyD88/- male grafts on days 0,7 , and 14 after transplantation (on day 14 , MyD88 $8^{+/+}$ $\mathrm{CD}^{-} \mathrm{CD} 11 \mathrm{c}^{+}$vs. MyD88 ${ }^{-/-} \mathrm{CD} 40^{-} \mathrm{CD} 11 \mathrm{c}^{+} \mathrm{P}=0.0002 ; \mathrm{MyD}^{-1 /+}$

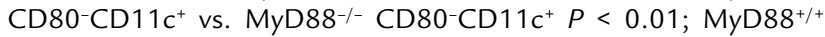
$\mathrm{CD}^{-} 6^{-} \mathrm{CD} 11 \mathrm{c}^{+}$vs. MyD88 $\left.{ }^{-/-} \mathrm{CD} 86^{-} \mathrm{CD} 11 \mathrm{c}^{+} P<0.01\right)$. (c) Analysis of the number of mature DCs in the draining lymph nodes in different transplant combinations on day 14 after transplantation. The presence of WT APCs in either the donor or the recipient partially restored the number of mature DCs present in the draining lymph nodes when compared with the $\mathrm{MyD} 88^{+/+} \rightarrow \mathrm{MyD} 88^{+/+}$transplant

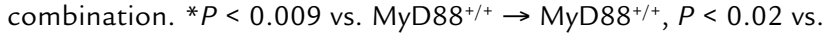

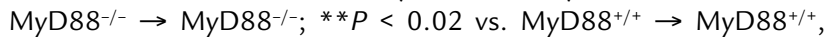
$P<0.006$ vs. MyD88 $8^{-/} \rightarrow \mathrm{MyD} 88^{-1-}$.

\section{Figure 1}

(a) Rejection of HY-incompatible skin allografts depends on the presence of MyD88. Eight out of nine MyD88-/- female recipients were unable to reject MyD88/- male donor skin grafts (filled circles), whereas WT littermate controls (filled squares) manifested a median allograft survival time of 16 days $(P=0.00001)$. TLR2 ${ }^{-/-}$(triangles) and $\mathrm{ICE}^{-/-}$(open squares) recipients demonstrated a modest but significant delay in allograft rejection $(P=0.02$ and $P=0.01$, respectively), whereas allograft rejection in TLR4 $4^{-/}$recipients (diamonds) was not different from that in controls $(P=0.13)$ despite the presence of one outlier that did not reject its allograft. (b) Adoptive transfer of primed WT spleen cells restores allograft rejection. MyD88 $/-$ female recipients infused in vivo with $4 \times 10^{7}$ primed WT spleen cells at the time of transplantation (squares) rejected their HY-incompatible allografts. $P=0.00003$ vs. a control group (diamonds) of female MyD88 $/ /$ recipients that were infused with primed male MyD88 ${ }^{-/-}$spleen cells.

was significantly inferior to that in $\mathrm{MyD} 88^{-/-}$recipients (TLR2 $^{-/-}$vs. $\mathrm{MyD}^{-/-} P=0.0001, \mathrm{ICE}^{-/-}$vs. $\mathrm{MyD} 88^{-/-}$ $P=0.0004)$. TLR $4 /-$ recipients were able to reject male allografts in a fashion similar to that of littermate controls, despite one TLR4 ${ }^{-/-}$recipient that manifested indefinite survival (TLR4 ${ }^{-/}$MST $=20$ days vs. littermate control MST $=16$ days, $P=0.13$ ) (Figure 1a). All syngeneic grafts were accepted indefinitely. These results demonstrate that rejection of HY-incompatible allografts depends on innate immune signaling via MyD88.

To determine whether MyD88 expression is important in the initiation phase of an alloimmune response (APC maturation and migration, and priming of T cells) or the effector phase (homing of effector $\mathrm{T}$ cells to the
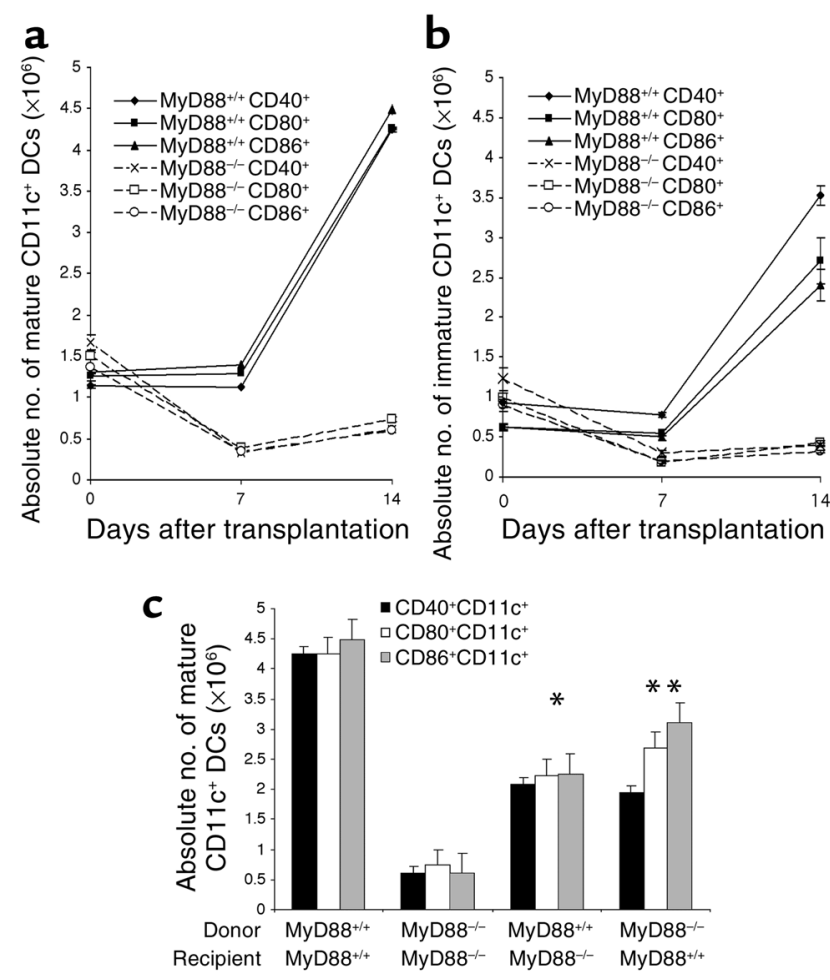

Transplant combinations 


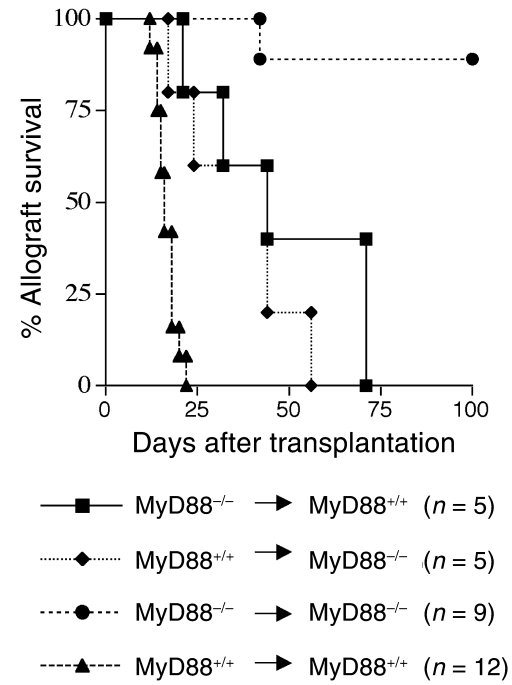

skin graft and its destruction), we investigated whether the absence of acute allograft rejection in the MyD88-recipients could be restored by the adoptive transfer of WT activated spleen cells. We infused mutant recipients at the time of skin transplantation with unfractionated spleen cells from WT female mice immunized with $\mathrm{MyD}^{+/+}$male spleen cells. A control group of MyD88-/- female recipients received spleen cells from female MyD88-/- mice immunized with male MyD88-/spleen cells. The rejection response was restored in the $\mathrm{MyD}^{-1-}$ recipients infused with primed, WT spleen cells, while the control group $(n=5)$ did not reject their allografts $\left(\mathrm{MyD}^{+/++}\right.$spleen cell-infused MST $=32$ days vs. MyD88 ${ }^{-/}$spleen cell-infused MST > 100 days, $P=0.00003$ ) (Figure 1b). Since activated WT spleen cells could restore rejection, this indicates that the defect in

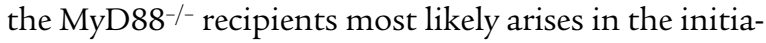
tion phase of the alloimmune response.

MyD88-/- recipients manifest reduced numbers of mature and immature DCs in the lymph nodes draining the allografts. As TLR ligation on DCs induces a maturation

\section{Figure 4}

MyD88/- mice have impaired adaptive immune responses to $\mathrm{HY}$ antigens. HY-specific tetramers were used to quantify the number of anti-HY CD8 ${ }^{+} \mathrm{T}$ cells in response to full-thickness male allografts. Fourteen days after transplantation, spleen cells were harvested from

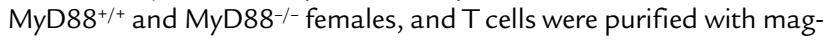
netic microbeads, stained with KCSRNRQYL HY tetramer $\left(H^{b}\right)$ conjugated to phycoerythrin and FITC-conjugated anti-CD8, and then analyzed by flow cytometry. (a) Representative flow cytometric plots of HY tetramer staining, showing base-line purified naive T cells in $\mathrm{MyD}^{+/++}$and $\mathrm{MyD} 88^{-/-}$mice; and plots of spleen cells from ${\mathrm{MyD} 88^{+/+} \text {and MyD88 }}^{-/-}$recipients of ${\mathrm{MyD} 88^{+/+} \text {and MyD88 }}^{-/-}$skin grafts, respectively, 14 days after transplantation, demonstrating a reduced number of $\mathrm{CD}^{+}$tetramer-positive cells in MyD88 ${ }^{-/-}$recipients. Positive control from an $\mathrm{HY}$ transgenic animal and specificity control using KAVYNFATC HD $^{\text {b }}$ tetramer are shown. (b) Histogram plot of pooled data from three experiments demonstrating that

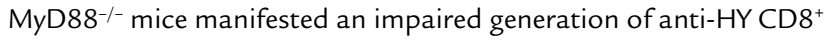
$\mathrm{T}$ cells in response to transplantation, compared with WT mice $(1.52 \%$ vs. $15.79 \%, P=0.01)$.

\section{Figure 3}

Restoration of allograft rejection by provision of either WT donor or recipient APCs. To restore WT recipient or donor APCs, WT females were transplanted with skin from male MyD88-1- donors (squares) or MyD88-1- females were transplanted with WT male skin (diamonds). All recipients in both groups consequently rejected their grafts with significantly inferior survival times versus $\mathrm{MyD} 88^{-/-}$recipients of MyD88-/- male grafts (circles) $(P=0.001$ in both cases). However, survival times were significantly longer than those of female MyD88 $8^{+/+}$ recipients of male $\mathrm{MyD} 88^{+/+}$allografts (triangles) $(P=0.001 \mathrm{vs}$.

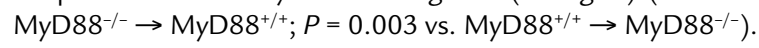

program that leads to initiation of an adaptive immune response, we next investigated whether defects in DC maturation occurred in MyD88-/female recipients of male MyD88 $/ /$ allografts. We quantified the numbers of mature DCs in the draining lymph nodes of transplant recipients during the first 2 weeks after transplantation. Lymph nodes

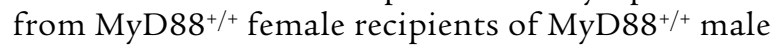
allografts and $\mathrm{MyD} 88^{-/-}$female recipients of male

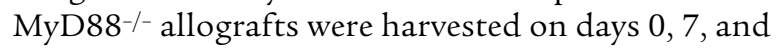
14 after transplantation. They were then stained for the DC-specific integrin receptor CD11c, and costimulatory maturation markers CD80, CD86, and $\mathrm{CD} 40 .{\mathrm{MyD} 88^{+/+}}$female recipients demonstrated a significant increase in the absolute number of mature DCs $\left(\mathrm{CD} 40^{+} \mathrm{CD} 11 \mathrm{c}^{+}, \mathrm{CD} 80^{+} \mathrm{CD} 11 \mathrm{c}^{+}\right.$, and $\left.\mathrm{CD}^{2} 6^{+} \mathrm{CD} 11 \mathrm{c}^{+} \mathrm{DCs}\right)$ on day 14 after transplantation (day 7 vs. day $14, P=0.001$ ) (Figure $2 a$ ). In contrast,

a

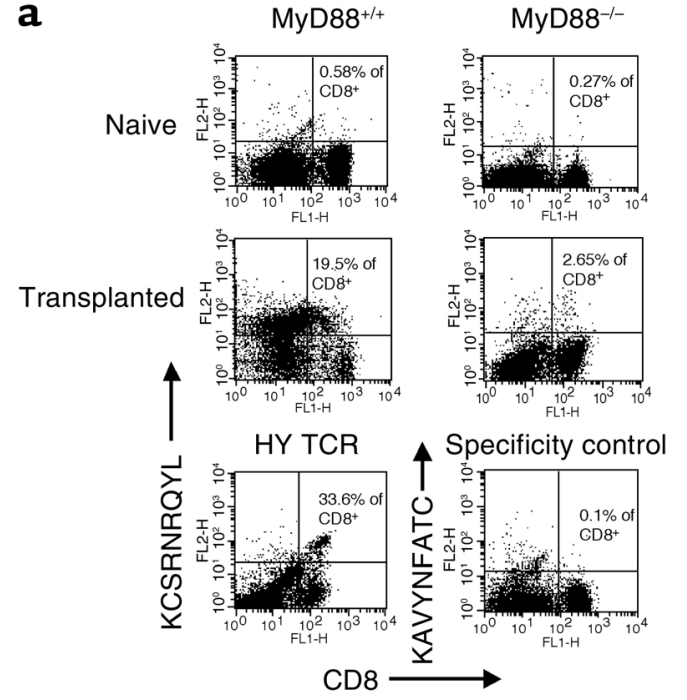

b

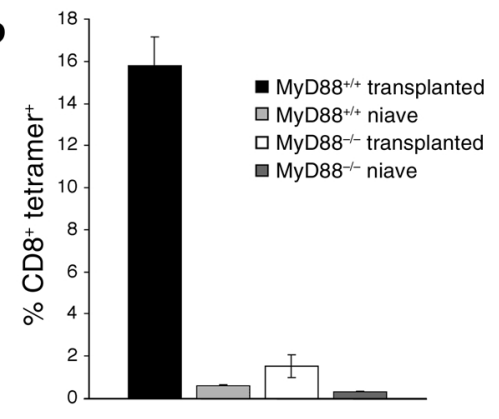



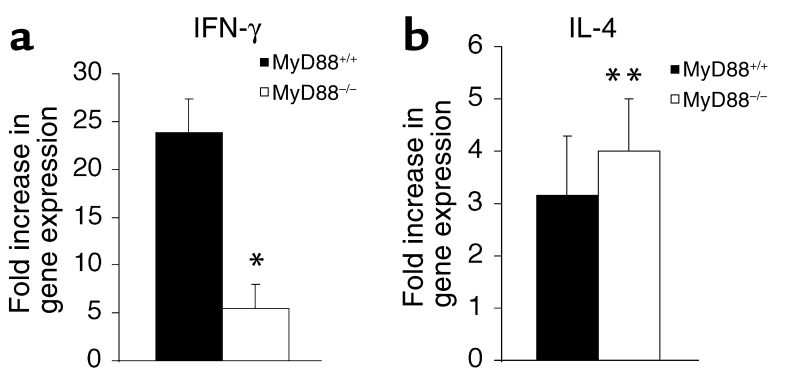

\section{Figure 5}

Impaired Th1 immune but intact Th2 immune responses in

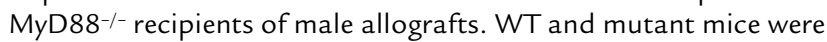
grafted with corresponding male allografts, and draining lymph nodes were isolated 14 days after transplantation. Gene expression for IFN- $\gamma(\mathbf{a})$ and IL-4 (b) was quantified using real-time PCR and reported as fold increase compared with that of naive mice in each group. The results show that in comparison to WT counterparts, MyD88 $/-$ recipients had impaired upregulation of IFN- $\gamma$ gene expression (5.5-fold vs. 23-fold increase, $\left.{ }^{*} P=0.0002\right)$. However, IL-4 gene expression was equally well expressed (4.0-fold vs. 3.14fold increase, $\left.{ }^{*} P=0.6\right)$. Experiments were repeated in triplicate.

despite having similar numbers of mature DCs on day $0, \mathrm{MyD} 88^{-/-}$recipients were not able to increase the absolute numbers of mature DCs during the first 2 weeks after transplantation (Figure 2a). The differences between $\mathrm{MyD}^{+/+}$and MyD88-/- recipients were significant on days 7 and 14 after transplantation $(P=0.0001)$, with the greatest difference noted at 14 days. Furthermore, MyD88-/- recipients manifested a failure to accumulate immature DCs on day 14 after transplantation (Figure 2b), indicating that deficient MyD88 signaling is important for DC migration in response to transplantation. Finally, the defect in accumulation of mature DCs was partially restored by either transplantation of a WT male donor onto a mutant female recipient $\left(\mathrm{MyD}^{+/+} \rightarrow \mathrm{MyD}^{+/ 8^{-/}}\right.$) or the reverse $\left(\mathrm{MyD}^{-/-} \rightarrow \mathrm{MyD}^{-/ /+}\right.$) (Figure 2c). The

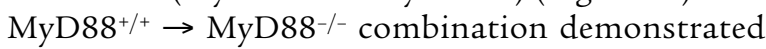
that WT APCs present in donor tissue (the allograft) were able to mature in the MyD88 $/-$ recipient. These results demonstrate that accumulation of mature and immature DCs in local lymph nodes in response to transplantation is impaired when TLR signaling through MyD88 is absent, and they provide further evidence that the defect in MyD88--- female recipients of male MyD88 $8^{-/}$allografts arises in the initiation phase of the alloimmune response.

$M y D 88$ expression in either the graft or the recipient is sufficient for rejection of HY-incompatible skin allografts. As MyD88 ${ }^{-1-}$ DCs manifested impaired migration and maturation in response to transplantation, we investigated whether providing either WT recipient APCs or WT donor passenger APCs could restore allograft rejection. We transplanted ${\mathrm{MyD} 88^{+/+}}$female recipients with male $\mathrm{MyD} 88^{-/-}$skin grafts and, conversely, MyD88-/- female recipients with male $\mathrm{MyD}^{-1 /+}$ grafts. The results show that when either WT recipient or WT donor APCs were present, all recipients rejected their allografts $\left(\mathrm{MyD} 88^{-/-} \rightarrow \mathrm{MyD}^{+/+}\right.$and

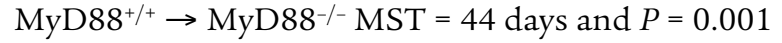

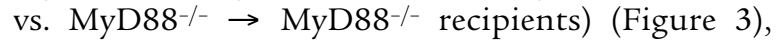
although the rejection was significantly delayed in both groups compared with WT littermate controls $\left(\mathrm{MyD}^{2} 8^{-/-} \rightarrow \mathrm{MyD}^{-/ /+} \mathrm{P}=0.001\right.$ and $\mathrm{MyD} 88^{+/+} \rightarrow$ \left.${\mathrm{MyD} 88^{-/-} P=0.003 \text { vs. MyD88 }}^{+/+} \rightarrow \mathrm{MyD}^{+/+}\right)$. Hence, the presence of MyD88 signaling in either donor skin or recipient is sufficient for rejection of HY-incompatible skin allografts. Furthermore, these results indicate that effector function in the $\mathrm{MyD} 88^{-/-}$recipient was adequate to induce allograft rejection once the alloimmune response was initiated by WT APCs (supplied via a WT allograft).

Impaired adaptive immunity in $M y D 88^{-/-}$recipients of HY-mismatched allografts. Since it has been shown that adaptive immune responses are controlled by TLR activation $(6,7)$, we investigated whether MyD88-/female recipients manifest reduced numbers of antiHY graft-reactive $T$ cells after transplantation. We used a specific KCSRNRQYL anti-HY tetramer to quantify $\mathrm{CD}^{+}$antigraft $\mathrm{T}$ cells in WT and $\mathrm{MyD} 88^{-/-}$ recipients of male WT and MyD88 ${ }^{-/-}$skin grafts. Previous studies have demonstrated that this tetramer detects anti-HY $\mathrm{CD}^{+}$cells in the spleens of female recipients of male allografts and that the peptide sequence accelerates rejection of male allografts (21, 22). As DC maturation in the mutants was maximally impaired on day 14 after transplantation, we quantified graft-reactive $\mathrm{T}$ cells at this time point. Spleen cells from $\mathrm{MyD}^{-1-}$ and WT recipients were harvested, T cell-purified, and stained with phycoerythrinconjugated KCSRNRQYL anti-HY tetramer and FITC-conjugated anti-CD8. The results show that MyD88-- recipients had a reduced proportion of $\mathrm{CD}^{+}$cells that were tetramer-positive (graft-reactive) in comparison with WT recipients $(1.52 \%$ vs. $15.79 \%$, $P=0.01$ ) (Figure 4). These results indicate that activation of the adaptive immune system is impaired in MyD88 $/-$ recipients, providing evidence that innate immune recognition is required to launch an adaptive immune response to a solid-organ allograft.

$M y D 88^{-1-}$ recipients demonstrate reduced expression of the Th1 proinflammatory cytokine IFN- $\gamma$ but intact Th2 cytokine IL-4. TLR ligation in response to antigenic stimulation has been shown to increase Th1 immunity and secretion of IFN- $\gamma$ without influencing Th2 immune responses $(7,23)$. We investigated this in our model by harvesting draining lymph nodes on day 14 after transplantation in mutant and WT female recipients of mutant and WT allografts, respectively. We then measured IFN- $\gamma$ and IL-4 gene expression by real-time PCR and compared it with that of corresponding naive animals from each group. MyD88-/- recipients had reduced IFN- $\gamma$ expression compared with ${\mathrm{MyD} 88^{+/+}}^{+}$mice (5.5- vs. 23-fold increase, $P=0.0002$ ), but IL-4 expression was equally well expressed in both groups (results within each group are normalized to naive lymph nodes) (Figure 5). 
These results demonstrate that Th1, but not Th2, immune responses are impaired in $\mathrm{MyD}^{-/-}$recipients in response to HY-incompatible allografts.

\section{Discussion}

We have shown here that mice with targeted disruption of the TLR signal adaptor MyD88 were unable to reject HY-incompatible allografts. Furthermore, we have demonstrated that the rejection response could be restored by infusion of primed WT spleen cells or by provision of either WT donor or recipient APCs. We present evidence that the inability to reject allografts in mutant mice was associated with a reduced number of mature and immature DCs in the draining lymph nodes of allografts during the first 2 weeks after transplantation, and with impaired generation of antigraft $T$ cells. Additionally, mutant mice demonstrated impaired Th1 immunity to the allograft that correlated with an inability to reject HY-incompatible allografts, a well-established model of in vivo Th1driven immunity (24-26). Therefore, our data provide evidence that adaptive alloimmunity is controlled by the innate immune system. These findings are compatible with previous work investigating the role of TLRs in the recognition of microorganisms. Specifically, the work of Schnare et al., using MyD88 mutants and caspase- 1 controls as we did, demonstrated that $\mathrm{MyD}^{-/-}$mice manifested an impairment in DC maturation and secretion of IFN- $\gamma$ in response to microbial antigens (7). However, there have been no prior reports investigating the role of TLRs in solid-organ transplantation.

To date, at least ten TLRs have been discovered. These are germline-encoded receptors, the best known of which are TLR2 and TLR4. TLR2, by forming heterodimers with TLR1 or TLR6, recognizes peptidoglycan on Gram-positive bacteria, bacterial lipoproteins, and glycosylphosphatidylinositol lipids from $T$. cruzi. TLR4 forms a heterodimer with MD2 and CD14 and is critical for recognizing LPS, a cell wall component of Gram-negative bacteria (6). TLR5 is activated by bacterial flagellin, and TLR3 by double-stranded RNA (6). Regardless of which TLR is activated, TLR signal transduction follows a uniform path through the signal adaptor MyD88, leading to nuclear translocation of NF- $\mathrm{\kappa B}$ and increased production of proinflammatory cytokines and expression of costimulatory molecules $(3,7)$. Previous studies have demonstrated that signaling via TLR4 can induce DCs to mature in an MyD88-independent manner (27). Subsequently, others have identified TIRAP/MAL as an alternate signal adaptor protein that is used in TLR signaling (28, 29). Recent work has demonstrated that TLR2, TLR4, and TLR2-TLR6 and TLR2-TLR1 heterodimers can signal via TIRAP $(30,31)$, although TIRAP does not appear to be responsible for the MyD88-independent pathway signaling that occurs via TLR2 and TLR4 (30). Since TLR $4^{-/-}$recipients did not manifest a delay in allograft rejection, and, in the absence of MyD88 signaling, allograft rejection was essentially abolished, we do not believe that the MyD88-independent pathway plays a critical role in our model. However, we cannot exclude the possibility that the modest delay in allograft rejection in TLR2 ${ }^{-/-}$recipients is mediated by signaling via TIRAP/MAL and MyD88. Our work therefore demonstrates that TLR signaling via MyD88 is not restricted to infections but can be initiated by solid-organ transplantation. It should be noted that we cannot exclude the possibility that a closely linked 129 gene could have impacted our results.

The self/non-self model of discrimination proposes that TLRs are used to distinguish self from foreign antigens (4). In this model, PRRs, in this case TLRs, can recognize specific motifs on microorganisms, known as PAMPs. PAMPs themselves are not virulence factors that can otherwise easily mutate and evade detection from the immune system; rather, they have essential housekeeping functions common to a broad range of microorganisms. Hence, all Gram-negative bacteria have the ability, whether they are virulent or not, to activate signals via the TLR4 pathway by virtue of LPS in their cell walls that induces maturation and migration of DCs. The mature DCs, by secreting proinflammatory cytokines and expressing costimulatory molecules, can then "switch on" adaptive immunity by migrating to local lymph nodes and communicating with naive $T$ cells, leading to the generation of effector $\mathrm{T}$ cells. Hence the self/non-self model argues that innate immune recognition of invading pathogens allows optimal activation of adaptive immunity. However, this model has problems dealing with allografts that may not possess obvious PAMPs disparate from those of the host.

Our study has demonstrated that TLR, IL-1, and IL-18 signaling via MyD88 is critical for the rejection of HY-incompatible allografts, indicating that TLRs may be activated by endogenous ligands and not solely by "traditional" PAMPs. Our work does not address what ligand on the allograft is involved, but other investigators have suggested that TLRs can be activated by a variety of ligands, including surfactant, heat shock protein, heparan sulfate, and the contents of necrotic cells (10-13). An alternative explanation is that contaminating microorganisms on the allograft stimulate TLRs using "traditional" PRRs and PAMPs. Indeed, there are several reports that infectious agents, mainly viruses, can modify acute and chronic rejection in experimental and clinical transplantation (32-35). In addition, a recent article has demonstrated that LPS expression is increased in reperfusion injury in a rat model of hepatic transplantation (36). If LPS recognition by TLR4 were a major mechanism of allograft rejection in our model, one would have expected TLR4 ${ }^{-/}$recipients to have manifested a delay in allograft rejection, which was not the case. However, we cannot exclude the possibility that other bacterial motifs are important. 
Our work demonstrates that when DC maturation occurs through intact MyD88 signaling, maximal migration to the lymph nodes draining the allograft occurs in the second week after transplantation. This agrees with a previous study showing that maximal emigration of DCs from skin allografts begins 7 days after transplantation (37). Our data show the importance of TLRs for DC maturation and migration, since MyD88-/- recipients of mutant allografts manifested impaired accumulation of mature and immature DCs in the draining lymph nodes after transplantation. This accumulation was partially restored by either a donor or a recipient that was not MyD88-deficient and provides an explanation as to why these in vivo transplant combinations restored allograft rejection in a delayed manner. Our data also provide evidence that MyD88 signaling is important in the initiation phase of an alloimmune response and are compatible with previous work demonstrating the importance of TLRs for DC maturation in response to infectious agents (7). Since it has been shown that DCs must acquire a mature phenotype for effective migration $(38,39)$, our work suggests that the failure of $\mathrm{MyD}^{-/-}$recipients to accumulate DCs is likely due to aberrant DC maturation and subsequent trafficking. Alternatively, APCs, for example, monocytes and macrophages, that are known to express TLRs may also be involved in our model. In addition, APCs may be activated and matured indirectly via secreted cytokines (for example TNF- $\alpha$ and IL-1) from other cell types, including those of nonhematopoietic origin (for example, donor and recipient endothelial cells).

It is likely that more than one TLR is involved in allograft rejection, since, in the absence of TLR2, recipients had only a modestly delayed time to rejection. In addition, a non-TLR MyD88-dependent IL-1/IL-18 pathway plays a role, as caspase-1/-/ recipients also manifested a modest delay in allograft survival. This is not surprising given the proinflammatory nature of IL-1 and its role in upregulating adhesion molecules and acute-phase proteins (40). However, since caspase $-1^{-/-}$recipients manifested a large and significantly inferior allograft survival time in comparison to $\mathrm{MyD} 88^{-/-}$recipients, and since there are no other known receptors besides TLRs that utilize MyD88, TLRs must be involved in our model. Still, it is possible that the severely compromised allograft rejection in $\mathrm{MyD} 88^{-/-}$recipients results from impairment of both TLR and IL-1/IL-18 pathways. It is unclear at this point whether the difference demonstrated between $\mathrm{MyD}^{-/-}$and $\mathrm{ICE}^{-/-}$recipients results solely from TLR2 signaling, although this would appear unlikely given the very modest prolongation in allograft survival observed in TLR2-/recipients. As at least ten mammalian TLRs have been discovered, it is likely that the interactions among an allograft, TLRs, IL-1, and IL-18 are complex, involving multiple ligands and several receptors that act alone or in concert.
The immune response was not abolished in the

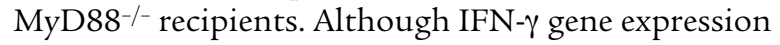
was impaired, IL-4 was intact, indicating a functional Th2 response that is in agreement with findings in a nontransplant setting (7). There are several experimental models in which allograft rejection can be mediated via eosinophils driven by Th2 cytokines (26). This could potentially explain why one of the MyD88-/recipients was able to reject its allograft. Furthermore, it has been postulated that Th2 responses and TLR responses may occur by a different set of PAMPs (7).

Future studies will be required to determine whether functional MyD88 signaling is important in fully MHC-mismatched allograft rejection of skin and vascular organs. Since we observed intact Th2 cytokine expression in the absence of MyD88, it is difficult to predict whether the MyD88 pathway is required for the rejection of fully $\mathrm{MHC}$-mismatched organs, because the rejection of such organs can be mediated by Th2 immunity (26). Nevertheless, our findings, using a wellcharacterized transplant model (41-44), demonstrate for the first time to our knowledge that MyD88 signaling is critical for allograft rejection. This is an important finding, since in vivo investigation of TLRs has predominantly involved infectious models.

In conclusion, by using an HY-incompatible transplant model, we have demonstrated that rejection of solid-organ transplants is dependent on signaling via MyD88, underscoring the importance of innate immunity in allograft rejection. This work demonstrates that the TLR signal adaptor protein MyD88 can be activated in the setting of solid-organ transplantation and not solely by infections.

\section{Acknowledgments}

We would like to thank Ira Melman (Yale University) for help with DC flow cytometry and Ruslan Medzhitov (Yale University) for helpful comments at the initiation of the project. We would also like to thank Seiyu Hosono (Molecular Staging Inc., New Haven, Connecticut, USA) for use of the ABI 7700 real-time PCR thermocycler. The work was supported by NIH grant KO8 AI1732 to D.R. Goldstein.

1. Khush, R.S., Leulier, F., and Lemaitre, B. 2002. Immunology: Enhanced: Pathogen Surveillance: the flies have it. Science. 296:273-275.

2. Medzhitov, R., Preston-Hurlburt, P., and Janeway, C.A., Jr. 1997. A human homologue of the Drosophila Toll protein signals activation of adaptive immunity. Nature. 388:394-397.

3. Akira, S., Takeda, K., and Kaisho, T. 2001. Toll-like receptors: critical proteins linking innate and acquired immunity. Nat. Immunol. 2:675-680.

4. Medzhitov, R., and Janeway, C.A., Jr. 2002. Decoding the patterns of self and nonself by the innate immune system. Science. 296:298-300.

5. Barton, G.M., and Medzhitov, R. 2002. Control of adaptive immune responses by Toll-like receptors. Curr. Opin. Immunol. 14:380-383.

6. Medzhitov, R. 2001. Toll-like receptors and innate immunity. Nat. Rev. Immunol. 1:135-145.

7. Schnare, M., et al. 2001. Toll-like receptors control activation of adaptive immune responses. Nat. Immunol. 2:947-950.

8. Janeway, C.A., Jr. 1989. Approaching the asymptote? Evolution and revolution in immunology. Cold Spring Harb. Symp. Quant. Biol. 54:1-13.

9. Medzhitov, R., and Janeway, C.A., Jr. 1997. Innate immunity: the virtues of a nonclonal system of recognition. Cell. 91:295-298.

10. Johnson, G.B., Brunn, G.J., Kodaira, Y., and Platt, J.L. 2002. Receptor- 
mediated monitoring of tissue well-being via detection of soluble heparan sulfate by Toll-like receptor 4. J. Immunol. 168:5233-5239.

11. Li, M., et al. 2001. An essential role of the NF-kappa B/Toll-like receptor pathway in induction of inflammatory and tissue-repair gene expression by necrotic cells. J. Immunol. 166:7128-7135.

12. Ohashi, K., Burkart, V., Flohe, S., and Kolb, H. 2000. Cutting edge: heat shock protein 60 is a putative endogenous ligand of the toll-like receptor-4 complex. J. Immunol. 164:558-561.

13. Guillot, L., et al. 2002. Cutting edge: the immunostimulatory activity of the lung surfactant protein-A involves Toll-like receptor 4. J. Immunol. 168:5989-5992.

14. Pockley, A.G. 2001. Heat shock proteins, anti-heat shock protein reactivity and allograft rejection. Transplantation. 71:1503-1507.

15. Adachi, O., et al. 1998. Targeted disruption of the MyD88 gene results in loss of IL-1- and IL-18-mediated function. Immunity. 9:143-150.

16. Ghayur, T., et al. 1997. Caspase-1 processes IFN-gamma-inducing factor and regulates LPS-induced IFN-gamma production. Nature. 386:619-623.

17. Goldstein, D.R., et al. 2000. A differential requirement for CD8+ donor cells in the augmentation of allograft survival by posttransplantation administration of donor spleen cells and donor bone marrow cells. Transplantation. 70:1068-1073.

18. Xia, D., Sanders, A., Shah, M., Bickerstaff, A., and Orosz, C. 2001. Real-time polymerase chain reaction analysis reveals an evolution of cytokine mRNA production in allograft acceptor mice. Transplantation. 72:907-914.

19. Dong, C., Zhu, S., Wang, T., Yoon, W., and Goldschmidt-Clermont, P. 2002. Upregulation of PAI-1 is mediated through TGF-beta/Smad pathway in transplant arteriopathy. J. Heart Lung Transplant. 21:999-1008.

20. Lechler, R., Ng, W.F., and Steinman, R.M. 2001. Dendritic cells in transplantation: friend or foe? Immunity. 14:357-368.

21. Millrain, M., et al. 2001. Examination of HY response: T cell expansion, immunodominance, and cross-priming revealed by HY tetramer analysis. J. Immunol. 167:3756-3764.

22. Maile, R., et al. 2001. Antigen-specific modulation of an immune response by in vivo administration of soluble MHC class I tetramers. J. Immunol. 167:3708-3714.

23. Scanga, C.A., et al. 2002. Cutting edge: MyD88 is required for resistance to Toxoplasma gondii infection and regulates parasite-induced IL-12 production by dendritic cells. J. Immunol. 168:5997-6001.

24. Valujskikh, A., Lantz, O., Celli, S., Matzinger, P., and Heeger, P.S. 2002 Cross-primed CD8(+) T cells mediate graft rejection via a distinct effector pathway. Nat. Immunol. 3:844-851.

25. Valujskikh, A., Hartig, C., and Heeger, P.S. 2001. Indirectly primed CD8+ $\mathrm{T}$ cells are a prominent component of the allogeneic $\mathrm{T}$-cell repertoire after skin graft rejection in mice. Transplantation. 71:418-421.

26. Le Moine, A., Goldman, M., and Abramowicz, D. 2002. Multiple pathways to allograft rejection. Transplantation. 73:1373-1381.
27. Kaisho, T., Takeuchi, O., Kawai, T., Hoshino, K., and Akira, S. 2001 Endotoxin-induced maturation of MyD88-deficient dendritic cells. J. Immunol. 166:5688-5694.

28. Horng, T., Barton, G.M., and Medzhitov, R. 2001. TIRAP: an adapter molecule in the Toll signaling pathway. Nat. Immunol. 2:835-841.

29. Fitzgerald, K.A., et al. 2001. Mal (MyD88-adapter-like) is required for Toll-like receptor-4 signal transduction. Nature. 413:78-83.

30. Yamamoto, M., et al. 2002. Essential role for TIRAP in activation of the signalling cascade shared by TLR2 and TLR4. Nature. 420:324-329.

31. Horng, T., Barton, G.M., Flavell, R.A., and Medzhitov, R. 2002. The adaptor molecule TIRAP provides signalling specificity for Toll-like receptors. Nature. 420:329-333.

32. Vilchez, R.A., et al. 2002. Influenza virus infection in adult solid organ transplant recipients. Am. J. Transplant. 2:287-291.

33. Vilchez, R.A., et al. 2001. The epidemiology of parainfluenza virus infection in lung transplant recipients. Clin. Infect. Dis. 33:2004-2008.

34. Williams, M.A., et al. 2001. Characterization of virus-mediated inhibition of mixed chimerism and allospecific tolerance. J. Immunol. 167:4987-4995

35. Soderberg-Naucler, C., and Emery, V.C. 2001. Viral infections and their impact on chronic renal allograft dysfunction. Transplantation. 71(Suppl. 11):SS24-SS30.

36. Tsoulfas, G., et al. 2002. Activation of the lipopolysaccharide signaling pathway in hepatic transplantation preservation injury. Transplantation. 74:7-13.

37. Larsen, C.P., et al. 1990. Migration and maturation of Langerhans cells in skin transplants and explants. J. Exp. Med. 172:1483-1493.

38. Luster, A.D. 2002. The role of chemokines in linking innate and adaptive immunity. Curr. Opin. Immunol. 14:129-135.

39. Banchereau, J., and Steinman, R.M. 1998. Dendritic cells and the control of immunity. Nature. 392:245-252.

40. Dinarello, C.A. 1996. Biologic basis for interleukin-1 in disease. Blood. 87:2095-2147.

41. Mayer, T.G., Bhan, A.K., and Winn, H.J. 1988. Immunohistochemical analyses of skin graft rejection in mice. Kinetics of lymphocyte infiltration in grafts of limited immunogenetic disparity. Transplantation. 46:890-899.

42. Valujskikh, A., Matesic, D., and Heeger, P.S. 1999. Characterization and manipulation of $\mathrm{T}$ cell immunity to skin grafts expressing a transgenic minor antigen. Transplantation. 68:1029-1036.

43. Anderson, C.C., and Matzinger, P. 2001. Immunity or tolerance: opposite outcomes of microchimerism from skin grafts. Nat. Med. 7:80-87.

44. Inoue, Y., Konieczny, B.T., Wagener, M.E., McKenzie, A.N.J., and Lakkis, F.G. 2001. Failure to induce neonatal tolerance in mice that lack both IL-4 and IL-13 but not in those that lack IL-4 alone. J. Immunol. 167:1125-1128 